\title{
In Vitro Inhibitory Effect of Lercanidipine on Cholesterol Accumulation and Matrix Metalloproteinases Secretion by Macrophages
}

\author{
Monica Canavesi, PhD, * Novella Baldini, PhD, † Amedeo Leonardi, PhD, $\neq$ Giorgio Sironi, PhD, $\neq$ \\ Stefano Bellosta, PhD, * and Franco Bernini, PhD广
}

\begin{abstract}
Plaque rupture and thromboembolism play a major role in atherosclerotic acute syndrome. Experimental studies have demonstrated the potential direct anti-atherosclerotic effects of calcium antagonists. We investigated the in vitro effect of lercanidipine (REC $15 / 2375$ ), a third-generation, highly lipophilic calcium antagonist on cholesterol metabolism and matrix metalloproteinases secretion in macrophages, two functions that predispose plaques to rupture. Lercanidipine $\left(10^{-6}-10^{-5} \mathrm{M}\right)$ inhibited cholesterol esterification in macrophages and reduced cellular free and esterified cholesterol accumulation from acetylated LDL $(63 \%, 62 \%$ of control $P<0.05$, respectively). In addition, lercanidipine inhibited the release of metalloproteinases in the extracellular medium (50\% and 95\% inhibition at $10^{-5} \mathrm{M}$ for MMP-9 and MMP-2, respectively). Experiments performed with lercanidipine enantiomers or other dihydropyridine derivatives, endowed with different lipophilicity and affinity for calcium channels, indicated that the above effects could be related to the lipophilic, but not to the calcium channel blocking properties of these molecules. When cells, after exposure to the drug, were allowed to equilibrate, lercanidipine inhibitory action could be observed at initial concentrations as low as $10^{-9} \mathrm{M}$, which is the actual concentration range observed in plasma in clinical settings. In conclusion, our data indicate that lercanidipine may exert potent anti-atherosclerotic effects by inhibiting macrophage functions involved in plaque stability.
\end{abstract}

Key Words: atherosclerosis, calcium antagonists, macrophages, cholesterol esterification, metalloproteinases

(J Cardiovasc Pharmacol ${ }^{\mathrm{TM}}$ 2004;44:416-422)

Received for publication January 30, 2004; accepted June 30, 2004

From the *Department of Pharmacological Sciences, University of Milan, Milan, Italy; †Department of Pharmacological and Biological Sciences and Applied Chemistries, University of Parma, Italy; \$Pharmaceutical R\&D Recordati SpA, Milan, Italy.

This study was supported in part by grant No QLG1-1999-01007 from the European Commission to the consortium "Macrophage Function and Stability of the Atherosclerotic Plaque (MAFAPS)" as part of the Fifth Framework Program of the European Union and by a grant from Compagnia di San Paolo and the Italian Ministry of University and Research (MIUR-FIRST).

Reprints: Monica Canavesi, Department of Pharmacological Sciences, University of Milan, via Balzaretti, 9, Milan, Italy 20133 (e-mail: fulab@ unimi.it).

Copyright $\odot 2004$ by Lippincott Williams \& Wilkins
P harmacological control of atherosclerosis may be achieved by both controlling cardiovascular risk factors and by directly modulating major cellular processes involved in atheroma formation. The composition and vulnerability of plaque are major determinants of atherosclerosis complications, such as plaque disruption and superimposed thrombosis. ${ }^{1}$ Macrophages infiltrated in the lesion play a major role in determining its stability. These cells may accumulate esterified cholesterol in the atheromatous core and may weaken the fibrous cap by secreting specific metalloproteinases (MMPs) that degrade the extracellular matrix. ${ }^{2,3}$ The well-documented therapeutic efficacy of statins may at least in part be due to their ability to directly modulate these two macrophages functions. ${ }^{4,5}$ Dihydropyridine calcium antagonists (CAs) are among the first drugs showed to possess a direct anti-atherosclerotic activity in experimental models. ${ }^{6}$ However, clinical trials either did not or only weakly supported the relevance of this effect in humans. ${ }^{7,8}$ In our laboratory, we previously demonstrated that the lipophilic CA lacidipine, but not nifedipine, may inhibit AcylCoA:cholesterol Acyl Transferase (ACAT) in macrophages and consequently the intracellular cholesteryl esters formation, ${ }^{9}$ and may inhibit also the secretion of metalloproteinases. ${ }^{10}$ Moreover, a direct effect on the arterial wall was consistently observed in vivo in rabbits. ${ }^{9,11}$ The antiatherosclerotic effect of lacidipine appeared to be unrelated to calcium channel blockade or blood pressure reduction. ${ }^{9-11}$ Recently a large clinical trial with lacidipine, the European Lacidipine Study on Atherosclerosis (ELSA), has confirmed the anti-atherosclerotic activity of the drug in humans. The principal results showed that long-term antihypertensive treatment with lacidipine slowed down the progression of carotid intimamedia thickness (IMT) in hypertensive patients to a greater extent than treatment using the $\beta$-blocker atenolol. The greater anti-atherosclerotic action of lacidipine with respect to atenolol seemed to be independent of the blood pressure-lowering effect, as 24-hour ambulatory systolic and diastolic blood pressure reductions were slightly lower in lacidipine-treated than in atenolol-treated subjects. No significant difference between treatments was found in any cardiovascular events, although 
the relative risk for stroke, major cardiovascular events, and mortality showed a trend favoring lacidipine. ${ }^{12}$

Lercanidipine is a new calcium antagonist with high lipophilicity and a chiral center. Its pharmacokinetic and pharmacodynamic characteristics are similar to lacidipine and it is classified as a once-a-day CA. Lercanidipine and lacidipine long duration of action are controlled by the tissue cell membrane compartment from which they are slowly released once they have distributed throughout the patient's body. ${ }^{13}$ Therefore, these two lipophilic CA combine a relatively short plasma half-life, a gradual onset of action, and an intrinsically long duration of action. Lercanidipine has been previously shown to exert anti-atherosclerotic effects in rabbits; it reduced the hyperplasia and fatty streaks with a significant and dosedependent effect in the thoracic and abdominal aorta, but without affecting plasma cholesterol levels. This action was also observed with its R-enantiomer that has an affinity for calcium channels two orders of magnitude lower than lercanidipine. ${ }^{14}$

In the present study we investigated the effects of lercanidipine on cellular functions involved in plaque stability, by evaluating its ability to inhibit cholesterol esterification, AcLDL-derived cholesterol accumulation, and metalloproteinases secretion in mouse peritoneal macrophages in culture and in human monocytes.

\section{METHODS}

Dulbecco minimum essential medium (DMEM), Penicillin-Streptomycin (10000 IU/ml-10000 UG/ml), and fetal calf serum (FCS) were purchased from GIBCO BRL (Life Technologies, Milan, Italy). Essentially fatty acid-free bovine serum albumin (EFAF) was obtained from Sigma Chemical Company (St. Louis, MO). Cell culture wells $(35 \mathrm{~mm}$, Multidish Nunclon) were obtained from NUNC (Roskilde, Denmark).

\section{Cells}

Mouse peritoneal macrophages (MPM) were obtained by peritoneal lavage from mice (BALB/c, Charles River, Calco, Italy), 3 days after intraperitoneal injection of thioglycollate. Cells $\left(2-3 \times 10^{6}\right)$ were plated in $35-\mathrm{mm}$ wells with DMEM containing 10\% FCS. After 2 hours, the dishes were washed to eliminate unattached cells and maintained in DMEM plus 10\% FCS for 24 hours before use.

Circulating human monocytes were isolated from blood of healthy donors as previously described. ${ }^{5}$ The monocytes were collected, washed, resuspended in serum free DMEM, and plated at a density of $3 \times 10^{6}$ cells in a $35-\mathrm{mm}$ dish. After 2 hours, cell monolayers were washed twice and the adherent cells were incubated for 10 to 14 days with DMEM containing $10 \%$ human $\mathrm{AB}$ serum and insulin $8 \mu \mathrm{g} / \mathrm{ml}$, to allow for differentiation in macrophages.

Experiments were performed at $37^{\circ} \mathrm{C}$ in serum free DMEM containing $0.2 \%$ EFAF and the indicated concentra- tions of drugs, in presence or absence of phorbol esters [phor bol-12-myristate-13-acetate (PMA), $50 \mathrm{ng} / \mathrm{ml}$ ] or tumor necrosis factor- $\alpha$ (TNF- $\alpha, 20 \mathrm{ng} / \mathrm{ml})$ (Sigma Aldrich, St. Louis, MO).

Cellular protein content was measured according to Lowry et al. ${ }^{15}$ Cellular viability was assessed using the dimethylthiazoldiphenyltetrazolium bromide (MTT) assay as described. ${ }^{16}$ No cellular toxicity was observed at the drug concentrations tested.

\section{Lipoprotein Preparation}

Human LDL $(\mathrm{d}=1.019-1.063 \mathrm{~g} / \mathrm{ml})$ were isolated from plasma of healthy volunteers by sequential ultracentrifugation (Beckman L5-50, Palo Alto, CA). ${ }^{17}$ For acetylation, LDL were dialyzed against $0.15 \mathrm{M} \mathrm{NaCl}, \mathrm{pH} 7.4$, diluted with an equal volume of saturated $\mathrm{Na}$ acetate, and treated with acetic anhydride, according to Basu et al. ${ }^{18}$

AcLDL were radiolabeled with $\left[{ }^{3} \mathrm{H}\right]$-cholesteryl linoleate (Amersham, Buckinghamshire, UK) $\left(\left[^{3} \mathrm{H}\right]-\mathrm{CE}-\mathrm{AcLDL}\right)$ by incubation with serum containing the cholesteryl ester transfer protein. ${ }^{19}$ All lipoproteins were sterile filtered.

\section{Cholesterol Esterification Assay (ACAT Activity)}

Cells were incubated with the drug and AcLDL (50 $\mu \mathrm{g} / \mathrm{ml})$ as indicated. Cholesterol esterification was measured after addition of $\left[1-{ }^{14} \mathrm{C}\right]$ oleic acid $(0.68 \mu \mathrm{Ci} /$ sample $)$ complexed with bovine serum albumin during the last 2 hours of incubation and subsequent determination of radioactivity associated with cellular cholesteryl esters. ${ }^{20}$

At the end of incubation, cells were washed with phosphate-buffered saline (PBS) and lipids were extracted with hexane/isopropanol (3:2). The extracted lipids were separated by thin layer chromatography (TLC) (isooctane/diethyl ether/acetic acid, 75:25:2, vol/vol/vd). Cholesterol radioactivity in the spots was determined by liquid scintillation counting (Insta-Fluor, Packard, Groningen, The Netherlands).

\section{Intracellular Distribution of Lipoprotein-Derived Cholesterol}

Mouse peritoneal macrophages were incubated with lercanidipine for 30 hours and then with the drug and $\left[{ }^{3} \mathrm{H}\right]-\mathrm{CE}-$ AcLDL (30 $\mu \mathrm{g}$ protein $/ \mathrm{ml}$ ). The medium was subsequently discarded, cells were washed with PBS, and the lipids were extracted with hexane: isopropanol $(3: 2, \mathrm{vol} / \mathrm{vol})$. Free and esterified cholesterol were partitioned by TLC and the radioactivity of the spots was determined by liquid scintillation counting (Insta-Fluor, Packard, Groningen, The Netherlands).

\section{SDS Page Zymography}

At the end of the incubation, the conditioned media were collected and MMPs gelatinolytic capacity was evaluated as described previously. ${ }^{5}$ Briefly, samples underwent electrophoresis on polyacrylamide gels containing SDS and gelatin (1 $\mathrm{mg} / \mathrm{ml}$ ) under non-reducing conditions. After washes, the gels 
were incubated overnight at $37^{\circ} \mathrm{C}$ with gentle shaking in TRIS $50 \mathrm{mM} \mathrm{pH} 7.5$ containing $\mathrm{NaCl} 150 \mathrm{mM}, \mathrm{CaCl}_{2} 10 \mathrm{mM}, \mathrm{ZnCl}_{2}$ $1 \mu \mathrm{M}$, to activate the metalloproteinase ability to digest the substrate. At the end of the incubation, the gels were stained with a solution of Coomassie brilliant blue R-250 (SigmaAldrich, Milan, Italy). Clear zones against the blue background indicated the presence of proteinolytic activity. It has been described that, in this type of SDS-containing gel, the latent form of MMP-9, the pro-MMP-9, and the activated gelatinase develop gelatinolytic activity ${ }^{21}$; therefore, we used the word "activity" to indicate the potential total gelatinolytic capacity measured in the conditioned media. We have previously shown ${ }^{5}$ that, in our experimental conditions, the gelatinolytic capacity is due entirely to the $92-\mathrm{kDa}$ proMMP-9, as assessed by zymography and by Western blot analysis with a specific antibody against human MMP-9.

\section{ELISA}

The amount of secreted MMP-9 protein was quantified using the highly specific Biotrak ${ }^{\mathrm{TM}}$ Matrix Metalloproteinase-9 ELISA system (Amersham Pharmacia, Milan, Italy). The MMP-9 assay uses 2 antibodies directed against different epitopes of human MMP-9 and does not show detectable cross-reactivity with MMP-1, -2 , and -3 , and TIMP-1 and -2 (Amersham Pharmacia, Milan, Italy). Aliquots of conditioned media were analyzed as suggested by manufacturer.

\section{Statistical Analysis}

For quantitation of zymograms, densitometric scanning was performed using a system incorporating a video camera and a computer analysis package (NIH Image 1.52 image analysis software) as in reference 5 . Each experiment was performed at least 3 times with different preparations of cells. Results were normalized by cellular protein content and expressed as optical density (O.D.) units.

Data are expressed as mean $\pm \mathrm{SD}(\mathrm{SD})$. The results were analyzed by one way analysis of variance (ANOVA) for the comparison of multiple groups, followed by a Dunnet test.

\section{RESULTS}

Lercanidipine was evaluated for its effect on ACAT activity in mouse peritoneal macrophages. The results indicated that the drug was able to inhibit up to $90 \%$ in a concentrationdependent manner the formation of esterified cholesterol induced by AcLDL with an $\mathrm{IC}_{50}$ of $2.37 \mu \mathrm{mol} / \mathrm{l}$ (Fig. 1). We next evaluated the effect of lercanidipine on macrophage content of AcLDL-derived cholesterol. To this aim macrophages were incubated for 30 hours with lercanidipine, followed by an incubation with the drug and $\left[{ }^{3} \mathrm{H}\right] \mathrm{CE}-\mathrm{AcLDL}$ for an additional 18 hours. After the incubation period, cells were tested for their content of radioactive free, esterified, and total cholesterol. Results in Table 1 indicate that lercanidipine reduced both free and esterified cellular $\left[{ }^{3} \mathrm{H}\right]$ cholesterol, resulting in an overall

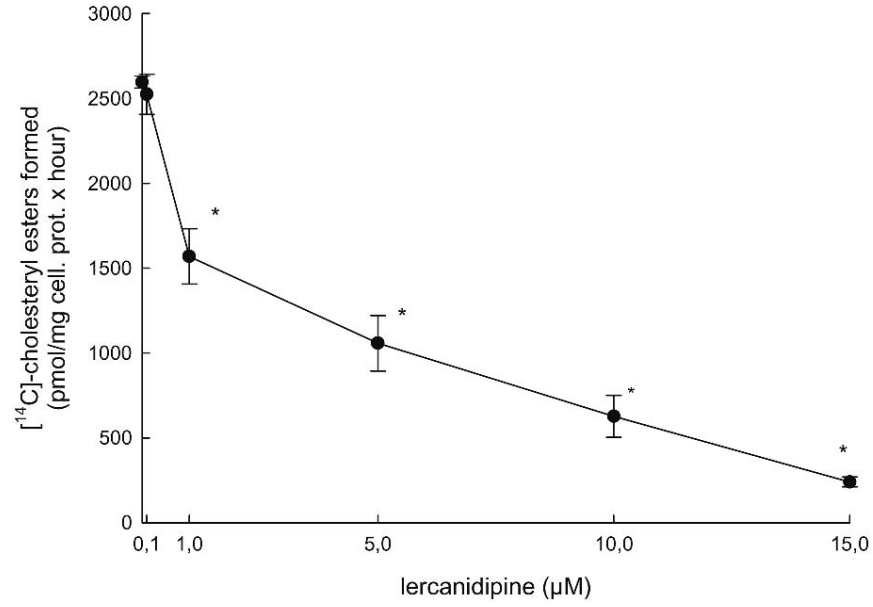

FIGURE 1. Effect of lercanidipine on cholesterol esterification in mouse macrophages. Cells were incubated for 24 hours in DMEM+EFAF $0.2 \%$ and lercanidipine, then with lercanidipine and AcLDL for 24 hours. In the last 2 hours $\left[{ }^{14} \mathrm{C}\right]$-oleic acid albumin complex was added for the determination of cholesterol esterification (ACAT activity). ${ }^{*} P<0.01$ vs control.

reduction in total AcLDL-derived cellular cholesterol content. To investigate whether the inhibitory effect of lercanidipine was related to its calcium antagonist property, we evaluated the effect on ACAT activity of lercanidipine enantiomers and of four 1,4 DHPs: Rec 15/2513, 15/2516, 15/2520, $15 / 2550$, in addition to the well-known dihydropyridine CA nitrendipine. These compounds differ or not in their ability to block calcium channels, lipophilicity, or the presence of ionizable amino group(s). Compounds with lower lipophilicity (15/2520 and nitrendipine) inhibited cholesterol esterification by less than $30 \%$; the molecules with higher lipophilicity, instead, exerted a more evident effect (40 to 95\%) (Table 2). No correlation was observed with compound affinities for calcium channels or with the presence of ionizable amino group.

We next evaluated the effect of lercanidipine on the secretion of MMP-9 by macrophages. MMP-9 is expressed by virtually all activated macrophages, and has been shown to be present in atherectomy materials from unstable angina. ${ }^{22}$ Mouse peritoneal macrophages treatment of 24 hours with lercanidipine significantly reduced, in a concentration-dependent manner, the gelatinolytic activity of MMP-9 secreted from 10 to $50 \%$, as assessed by gelatin zymography (Fig. 2). The two enantiomers reduced macrophage gelatinolytic activity to the same extent (Fig. 3). To better describe the base of this action, we measured lercanidipine effect on the gelatinolytic potential of MMP-9 already secreted. In this experimental condition, lercanidipine was ineffective (data not shown), suggesting that the reduced gelatinolytic activity measured by zymography is due to the inhibition of MMP-9 released by cells. To further confirm this, we measured directly the amount of 
TABLE 1. Effect of Lercanidipine on Cholesterol Distribution in Mouse Macrophages Incubated with $\left[{ }^{3} \mathrm{H}\right] \mathrm{CE}-\mathrm{AcLDL}$

\begin{tabular}{|c|c|c|c|}
\hline & Free Chol & $\begin{array}{c}\text { Esterified Chol } \\
\left(\text { dpm/mg prot. } \times 10^{3}\right)\end{array}$ & Total Chol \\
\hline Control & $22.2 \pm 3.1$ & $14.6 \pm 2.6$ & $45.8 \pm 1.8$ \\
\hline Lercanidipine $1 \mu \mathrm{M}$ & $16.3 \pm 2.9$ & $11.3 \pm 2.4$ & $33.7 \pm 1.5^{* *}$ \\
\hline Lercanidipine $10 \mu \mathrm{M}$ & $13.9 \pm 2.3^{*}$ & $9.1 \pm 1.8^{*}$ & $21.9 \pm 3.7^{* *}$ \\
\hline $\begin{array}{l}\text { (3-[decyldimethylsilyl]-N-[2-(4-methylphenyl)- } \\
\text { 1-phenylethyl] propanamide) } 2.15 \mu \mathrm{M}\end{array}$ & $22.3 \pm 4.5$ & $7.7 \pm 1.3^{*}$ & $37.5 \pm 5.3^{*}$ \\
\hline
\end{tabular}

MMP-9 released by human macrophages incubated with lercanidipine, using an ELISA assay. In this experiment we showed a reduction of the amount of MMP-9 protein actually released by cells into the culture medium (control $0.301 \pm 0.06$, lercanidipine $5 \mu \mathrm{M} 0.199 \pm 0.006 \mathrm{ng}$ MMP-9/ $\mu \mathrm{g}$ cellular protein; $P<0.01$ ).

In our experimental conditions, macrophages also secreted MMP-2, the constitutive form of the gelatinases. Lercanidipine also inhibited, in a concentration-dependent man ner, the gelatinolytic activity of MMP-2, from 20 to $95 \%$ (Fig. 2).

As reported in Table 3, the drug was still effective in activated murine macrophages, when metalloproteinases expression was stimulated by treatment with TNF- $\alpha$, or in human macrophages stimulated by treatment with phorbol esters.

The concentrations of lercanidipine active in all the above experiments were higher than its plasma concentrations observed in humans (in the range of $\mathrm{nM}$ ). Since lercanidipine is slowly released from the plasma membrane, we hypothesized that the drug could require some time to equilibrate within the intracellular pools where it could exert its inhibitory activity on macrophage functions. To investigate this aspect, cells were incubated with lercanidipine for 24 hours and then the drug was removed from cells. The evaluation of cholesterol esterification was performed 48 hours afterward. The results indicated that in these conditions lercanidipine not only maintained its inhibitory activity, but it was also more potent than what was observed with shorter experiment duration. In fact, a $60 \%$ inhibition of ACAT activity was seen at a concentration as low as $1 \mathrm{nM}$ (Fig. 4). At the same concentration we also observed an inhibition of metalloproteinases gelatinolytic activity (50\% and 65\% for MMP-9 and MMP-2, respectively; Fig. 5).

TABLE 2. Physico-Chemical Properties and Effect on Cholesterol Esterification of Nitrendipine, Lercanidipine, and Its Derivatives

\begin{tabular}{|c|c|c|c|c|c|c|}
\hline \multirow[b]{2}{*}{$\mathbf{R}$} & & \multirow[b]{2}{*}{$\begin{array}{c}\mathrm{Ca}++-C h a n n e l s \\
\text { Affinity } \\
\mathbf{K}_{\mathbf{i}} \text { BDG }(\mathbf{n M})\end{array}$} & \multirow[b]{2}{*}{$\begin{array}{l}\text { Lipophilicity } \\
\text { (Log K'w) }\end{array}$} & \multirow[b]{2}{*}{$\begin{array}{c}\text { Ionizable } \\
\text { Amino } \\
\text { Group(s) }\end{array}$} & \multicolumn{2}{|c|}{$\begin{array}{c}\text { ACAT } \\
\text { Activity in MPM }\end{array}$} \\
\hline & & & & & $\begin{array}{c}\text { \% of Inhibition } \\
(5 \mu \mathrm{M})\end{array}$ & $\begin{array}{l}\mathrm{IC}_{50} \\
(\mu \mathrm{M})\end{array}$ \\
\hline $\mathrm{C}\left(\mathrm{CH}_{3}\right)_{2} \mathrm{CH}_{2} \mathrm{NHCH}_{3}$ & $\operatorname{Rec} 15 / 2520$ & $\sim 1000$ & 3.60 & Yes & $25.9 \pm 4.75$ & N.C. \\
\hline $\mathrm{CH}_{2} \mathrm{CH}_{3}$ & Nitrendipine & 0.10 & 4.50 & No & $25.4 \pm 12.9$ & N.C. \\
\hline (*) $\mathrm{C}\left(\mathrm{CH}_{3}\right)_{2} \mathrm{CH}_{2} \mathrm{~N}\left(\mathrm{CH}_{3}\right) \mathrm{CH}_{2} \mathrm{CH}_{2} \mathrm{CH}\left(\mathrm{C}_{6} \mathrm{H}_{5}\right)_{2}$ & $\operatorname{Rec} 15 / 2513$ & $>1000$ & 7.40 & Yes & $39.6 \pm 7.28$ & 9.40 \\
\hline $\mathrm{C}\left(\mathrm{CH}_{3}\right)_{2} \mathrm{CH}_{2} \mathrm{~N}\left(\mathrm{CH}_{3}\right) \mathrm{CH}_{2} \mathrm{CH}_{2} \mathrm{CH}\left(\mathrm{C}_{6} \mathrm{H}_{5}\right)_{2}$ & (S)-lercanidipine & 0.11 & 7.35 & Yes & $47.6 \pm 0.71$ & 4.52 \\
\hline (**) $\mathrm{C}\left(\mathrm{CH}_{3}\right)_{2} \mathrm{CH}_{2} \mathrm{~N}\left(\mathrm{CH}_{3}\right) \mathrm{CH}_{2} \mathrm{CH}_{2} \mathrm{CH}\left(\mathrm{C}_{6} \mathrm{H}_{5}\right)_{2}$ & $\operatorname{Rec} 15 / 2516$ & 169.8 & 7.26 & Yes & $68.8 \pm 1.65$ & 3.54 \\
\hline $\mathrm{C}\left(\mathrm{CH}_{3}\right)_{2} \mathrm{CH}_{2} \mathrm{~N}\left(\mathrm{CH}_{3}\right) \mathrm{CH}_{2} \mathrm{CH}_{2} \mathrm{CH}\left(\mathrm{C}_{6} \mathrm{H}_{5}\right)_{2}$ & (R)-lercanidipine & 37.8 & 7.35 & Yes & $69.2 \pm 1.25$ & 1.88 \\
\hline $\mathrm{C}\left(\mathrm{CH}_{3}\right)_{2} \mathrm{CH}_{2} \mathrm{~N}\left(\mathrm{CH}_{3}\right) \mathrm{COCH}_{2} \mathrm{CH}\left(\mathrm{C}_{6} \mathrm{H}_{5}\right)_{2}$ & $\operatorname{Rec} 15 / 2550$ & 4.30 & 6.80 & No & $97.2 \pm 0.32$ & 0.81 \\
\hline
\end{tabular}

(*)Oxidized to pyridine ring; (**) Nitro group at position 4 of the phenyl ring.

$\mathrm{Ca}^{++}$-channels affinity values (evaluated as displacement of $\left[{ }^{3} \mathrm{H}\right]$ nitrendipine binding from rat brain membranes) and lipophilicity values have been purchased by Pharmaceutical R\&D Division, Recordati S.p.A., Milan, Italy. 


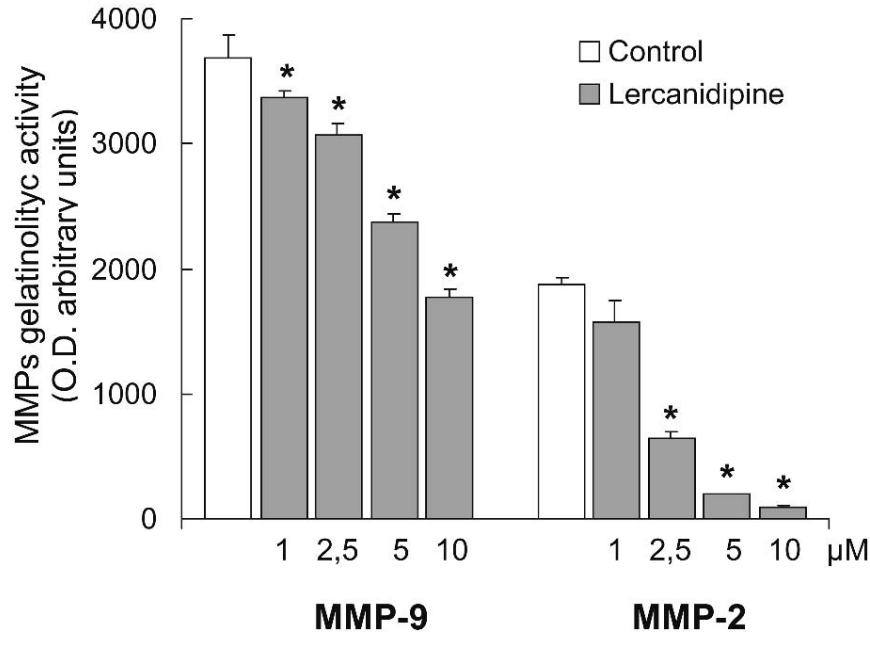

FIGURE 2. Effect of lercanidipine on MMPs gelatinolytic activity in mouse peritoneal macrophages. Cells were incubated for 24 hours in DMEM+EFAF $0.2 \%$ and lercanidipine. The gelatinolytic activity of MMP-9 and MMP-2 in the media was analyzed by gelatin zymography as described in Methods. ${ }^{*} P<$ 0.01 versus control.

\section{DISCUSSION}

Lercanidipine is a once-a-day calcium antagonist of the third generation. As reported for lacidipine, its long duration of action is due to its high lipophilicity. In the present study we showed that lercanidipine inhibited in a concentrationdependent manner cholesterol esterification in macrophages in culture. Similar to what was observed with lacidipine, this effect was observed also in a cell free homogenate (data not shown), confirming the ability of these compounds to directly interfere with the ACAT enzyme. Interestingly, lercanidipinetreated cells exposed to radioactive cholesteryl esters labeled AcLDL had a reduced content of both free and esterified ra-

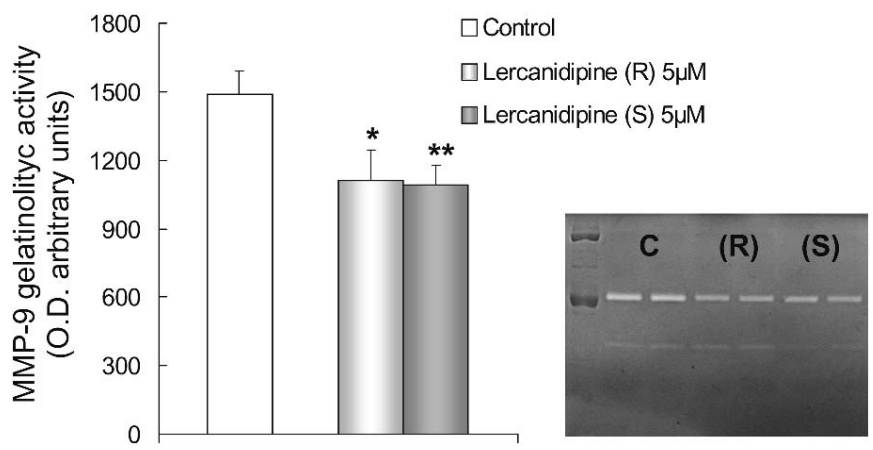

FIGURE 3. Effect of lercanidipine enantiomers on MMP-9 activity in mouse macrophages. Cells were incubated for 24 hours in DMEM+EFAF $0.2 \%$ and lercanidipine enantiomers. The gelatinolytic activity of MMP-9 in the media was analyzed by gelatin zymography. ${ }^{*} P<0.05 ;{ }^{* *} P<0.01$ versus control.

TABLE 3. Effect of Lercanidipine on MMP-9 Gelatinolytic Activity in Activated Macrophages

MMP-9 Gelatinolytic Activity (O.D./ $\mu$ g cell. protein)

Control

TNF- $\alpha 20 \mathrm{ng} / \mathrm{ml}$

TNF- $\alpha+$ Lercanidipine $1 \mu \mathrm{M}$

Control

PMA $50 \mathrm{ng} / \mathrm{ml}$

PMA + Lercanidipine $1 \mu \mathrm{M}$

Cells were incubated with lercanidipine and TNF- $\alpha$ or PMA for 24 hours. At the end of incubations gelatinolytic capacity was determined as described in Methods

$* P<0.01$ vs TNF- $\alpha$ or PMA alone.

dioactive cholesterol as compared with control. This result indicates that, despite the inhibitory action of lercanidipine on cholesterol esterification, the drug did not induce accumulation of cholesterol in the free fraction and cells exposed to the drug had a reduced ability to accumulate lipoprotein-derived cholesterol. This observation suggests that lercanidipine inhibitory effect on cellular cholesteryl ester formation is not entirely due to a direct ACAT inhibition, but additional mechanisms should be envisioned. This conclusion is supported by our observation that the addition of lercanidipine $\left(10^{-6} \mathrm{M}\right)$ to macrophages incubated with the specific ACAT inhibitor (3-

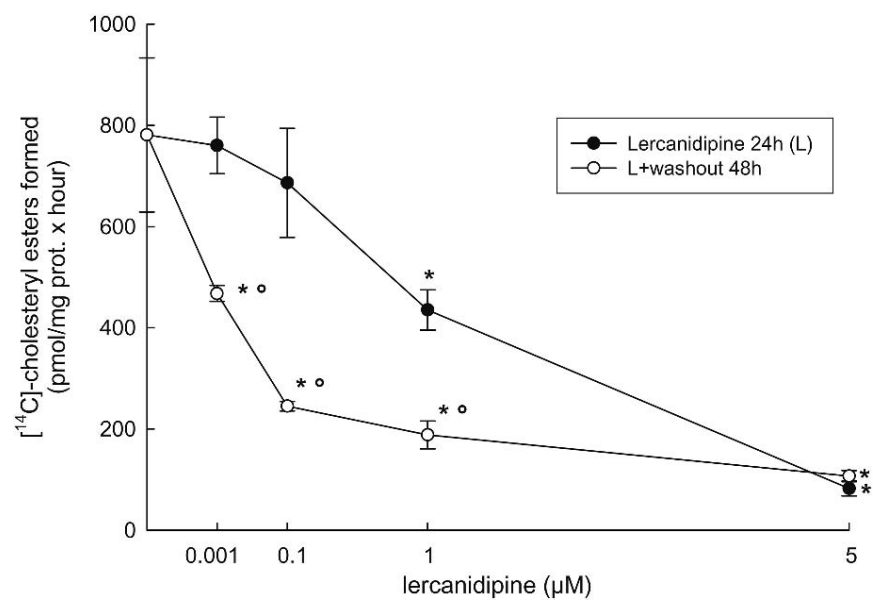

FIGURE 4. Effect of lercanidipine on cholesterol esterification in mouse macrophages after a period of washout. Cells were incubated for 24 hours in DMEM+EFAF $0.2 \%$ and lercanidipine; then with a drug-free medium for 48 hours. At the end all cells underwent 2 hours' incubation in presence of AcLDL and $\left[{ }^{14} \mathrm{C}\right]$-oleic acid albumin complex. ${ }^{*} P<0.01$ versus control; ${ }^{\circ} P<0.01$ versus lercanidipine alone. 


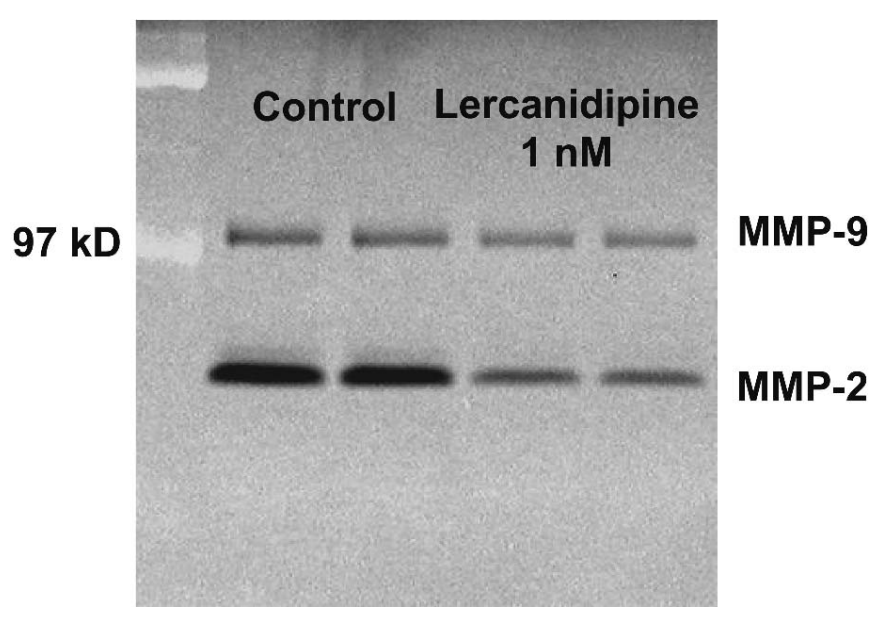

FIGURE 5. Effect of lercanidipine $1 \mathrm{nM}$ on MMPs gelatinolytic activity in mouse peritoneal macrophages after a period of washout. Cells were incubated for 24 hours in DMEM+EFAF $0.2 \%$ and lercanidipine $1 \mathrm{nM}$; then with a drug-free medium for 48 hours. The gelatinolytic activity of MMPs in the media was analyzed by gelatin zymography as described in Methods.

[decyldimethylsilyl]-N-[2-(4-methylphenyl)-1-phenylethyl] propanamide), leads to a further reduction of the cellular content of esterified cholesterol (from 5.5 to $3.7 \mathrm{dpm} / \mathrm{mg}$ prot. $\times$ $10^{3}$ ) without any increase in the free form. This effect could be explained by a possible increase in cholesterol efflux, a hypothesis supported by Robenek and Schmitz ${ }^{23}$ and, more recently, by Suzuki et al, ${ }^{24}$ who showed that calcium antagonists may increase cellular cholesterol release by different mechanisms.

We previously demonstrated that lacidipine, but not nifedipine, was able to inhibit cholesterol esterification in cell culture. ${ }^{9}$ This led to the hypothesis that the lipophilic properties of the former were involved in the drug inhibitory activity, independently of the affinity for calcium channels. In this study, we compared the effect on cholesterol esterification of the highly lipophilic lercanidipine with those of its two enantiomers and with a series of dydrophyridine derivatives with different degree of lipophilicity, affinity for the calcium channels, and containing or not a charged amino group. According to the previous hypothesis, both lercanidipine's enantiomers were active despite their different calcium channel blockade properties and the results suggested the involvement of lipophilicity as a necessary property for the inhibitory activity of cellular esterification, but not of calcium channel blockade. Since the activity of ACAT is sensitive to variations in the lipophilicity and composition of the biophase, ${ }^{25}$ the presence of the drug could alter the availability of the substrate for the enzyme. However, other structural characteristics of the molecules seem to be involved, as suggested by the differences in efficacy and potency among compounds in the high range of lipophilicity. In particular a stereospecific mechanism for
ACAT inhibition and the influence of ionizable amino group(s) could be envisioned.

Lercanidipine and both its enantiomers were also equally effective in reducing the gelatinolytic activity, measured in the media of treated macrophages, again suggesting an effect independent of calcium channels blockade. The effect of lercanidipine on gelatinolytic activity in the extracellular media is consequent to the decreased secretion of MMP-9 by cells. This observation is supported by the evidence that lercanidipine was not able to interfere directly with the gelatinolytic potential of media once the proteolytic enzymes have been already secreted and by the reduction of the actual amount of MMP-9 present in the incubation media, as measured by ELISA. This conclusion is entirely consistent with our previous observation obtained using lacidipine. ${ }^{10}$

Previous studies demonstrated that lacidipine and lercanidipine may exert direct effects on the arterial wall in rabbits at doses that give drug plasma concentrations similar to those observed in humans. ${ }^{9,11,14}$ Recently, Zanchetti et al ${ }^{12}$ demonstrated that lacidipine in men reduced IMT progression and plaque number to a greater extent than atenolol, despite a similar efficacy in blood pressure-lowering. The latter observations indicate that the direct effect of lipophilic CA on the arterial wall, demonstrated in the above-mentioned experimental models, may be relevant also to the clinical practice. However, the potential anti-atherosclerotic effects previously reported in cell cultures with lacidipine and, in similar experimental conditions (ie, 24 hours' incubation) with lercanidipine in the present study, occurred only at concentrations of the drugs that were 2 to 3 orders of magnitude higher then those measured in plasma, raising the question if the in vitro observations could be relevant to the in vivo effects obtained both in rabbits and in humans. Previously published data showed that lercanidipine reduction of the contractility of aorta strips persisted for several hours after removal of the compound from the bath. ${ }^{26}$ In addition we observed that lacidipine inhibitory effect on MMPs secretion by macrophages was maintained up to 48 hours after removal of the drug from the incubation media. ${ }^{10}$ In the present study we show that lercanidipine retains its ability to inhibit cholesterol esterification and MMP secretion in macrophages even at 2 days after wash out. In this experimental condition, the initial active concentrations of the drug were as low as $10^{-9} \mathrm{M}$, a concentration 2 to 3 orders of magnitude lower than in shorter experiments and similar to that observed in human plasma. ${ }^{27}$ This result suggests that once the drug has reached the plasma membrane, it slowly equilibrates within the intracellular pools where it may exert its inhibitory action on ACAT activity or MMP secretion. Therefore, lercanidipine may exert cellular effects in vitro at concentrations similar to those observed in clinical practice, as long as the drug has the time to reach the intracellular sites of action, a condition that occurs during therapy with this drug. 


\section{CONCLUSION}

In conclusion, our present study demonstrates that lercanidipine, at concentrations similar to those occurring in clinical practice, may inhibit in vitro macrophage functions involved in atherogenesis and plaque stability. These observations may in part explain the direct anti-atherosclerotic action of lipophilic calcium antagonists reported in animal models and in humans.

\section{REFERENCES}

1. Ross R. Atherosclerosis: an inflammatory disease. N Engl J Med. 1999; 340:115-126.

2. Libby P, Geng YJ, Aikawa M, et al. Macrophages and atherosclerotic plaque stability. Curr Opin Lipidol. 1996;7:330-335.

3. Lee RT, Libby P. The unstable atheroma. Arterioscler Thromb Vasc Biol. 1997; 17:1859-1867.

4. Bernini F, Scurati N, Bonfadini G, et al. HMG-CoA reductase inhibitors reduce acetyl LDL endocytosis in mouse peritoneal macrophages. Arterioscler Thromb Vasc Biol. 1995;15:1352-1358.

5. Bellosta S, Via D, Canavesi M, et al. HMG-CoA reductase inhibitors reduce MMP-9 secretion by macrophages. Arterioscler Thromb Vasc Biol. 1998;18:1671-1678.

6. Henry PD, Bentley KI. Suppression of atherogenesis in cholesterol-fed rabbits treated with nifedipine. J Clin Invest. 1981;68:1366-1369.

7. Jost S, Deckers J, Rafflenbeul W, et al. Features of the angiographic evaluation of the INTACT study. International Nifedipine Trial on Antiatherosclerotic Therapy. Cardiovasc Drugs Ther. 1990;4(Suppl 5):10371045.

8. Borhani NO, Mercuri M, Borhani PA, et al. Final outcome results of the Muticenter Isradipine Diuretic Atherosclerosis Study (MIDAS): a randomized controlled trial. JAMA. 1996;276:785-791

9. Bernini F, Canavesi M, Bernardini E, et al. Effect of lacidipine on cholesterol esterification: in vivo and in vitro studies. Br J Pharmacol. 1997; 122:1209-1215.

10. Bellosta S, Canavesi M, Favari E, et al. Lacidipine modulates the secretion of matrix metalloproteinase-9 by human macrophages. $J$ Pharmacol Exp Ther. 2001;296:736-743.

11. Soma MR, Donetti E, Seregni R, et al. Effect of lacidipine on fatty and proliferative lesions induced in hypercholesterolaemic rabbits. Br J Pharmacol. 1996;118:215-219.

12. Zanchetti A, Bond G, Hennig M, et al. Calcium antagonist lacidipine slows down progression of asymptomatic carotid atherosclerosis. Circulation. 2002;106:2422-2427.

13. Herbette LG, Vecchiarelli M, Sartani A, et al. Lercanidipine: Short plasma half-life, long duration of action and high cholesterol tolerance. Updated molecular model to rationalize its pharmacokinetic properties. Blood Press. 1998;2:10-17.

14. Soma MR, Natali M, Donetti E, et al. Effect of lercanidipine and its (R)enantiomer on atherosclerotic lesions induced in hypercholesterolemic rabbits. Br J Pharmacol. 1998;125:1471-1476.

15. Lowry OH, Rosebrough NJ, Farr AL, et al. Protein measurement with the Folin phenol reagent. J Biol Chem. 1951;193:265-275.

16. Mosmann T. Rapid colorimetric assay for cellular growth and survival: application to proliferation and cytotoxicity assay. J Immunol Methods. 1983;65:55-63.

17. Havel RJ, Rapaport E. Management of primary hyperlipidemia. $N$ Engl $J$ Med. 1995;332:1301-1307.

18. Basu SK, Goldstein JL, Anderson RGW, et al. Degradation of cationized low density lipoprotein and regulation of cholesterol metabolism in homozygous familial hypercholesterolemia fibroblasts. Proc Natl Acad Sci USA. 1976;73:3178-3182.

19. Nagelkerke JF, Van Berkel TJ. Rapid transport of fatty acids from rat liver endothelial to parenchymal cells after uptake of cholesteryl ester-labeled acetylated LDL. Biochim Biophys Acta. 1986;875:593-598.

20. Brown MS, Ho YK, Goldstein JL. The cholesteryl ester cycle in macrophage foam cells: continual hydrolysis and re-esterification of cytoplasmic cholesteryl esters. J Biol Chem. 1980;255:9344-9352.

21. Kleiner DE, Stetler-Stevenson WG. Quantitative zymography: detection of picogram quantities of gelatinases. Anal Biochem. 1994;218:325-329.

22. Brown DL, Hibbs MS, Kearney M, et al. Identification of 92-kDa gelatinase in human coronary atherosclerotic lesions: association of active enzyme synthesis with unstable angina. Circulation. 1995;91:2125-2131.

23. Robenek H, Schmitz G. $\mathrm{Ca}^{++}$antagonists and ACAT inhibitors promote cholesterol efflux from macrophages by different mechanism. II. Characterization of intracellular morphologic changes. Arteriosclerosis. 1988;8: $57-67$.

24. Suzuki S, Nishimaki-Mogami T, Tamehiro N, et al. Verapamil increases the apolipoprotein-mediated release of cellular cholesterol by induction of ABCA1 expression via liver X receptor-independent mechanism. Arterioscler Thromb Vasc Biol. 2004;24:519-525.

25. Suckling K, Stange EF. Role of acyl-CoA:cholesterol acyl-transferase in cellular cholesterol metabolism. J Lipid Res. 1985;26:647-671.

26. Leonardi A, Poggesi E, Taddei C, et al. In vitro calcium antagonist activity of lercanidipine and its enantiomers. J Cardiovasc Pharmacol. 1997;29: S10-S18.

27. Barchielli M, et al. Clinical pharmacokinetics of lercanidipine. J Cardiovasc Pharmacol. 1997;29:S1-S15. 